Pacific

Journal of

Mathematics

THIN POSITION OF A PAIR (3-MANIFOLD, 1-SUBMANIFOLD)

Chuichiro Hayashi and Koya Shimokawa

Volume $197 \quad$ No. 2

February 2001 


\title{
THIN POSITION OF A PAIR (3-MANIFOLD, 1-SUBMANIFOLD)
}

\author{
Chuichiro Hayashi And Koya Shimokawa
}

\begin{abstract}
We introduce the notions of Heegaard splittings and thin multiple Heegaard splittings of 1-submanifolds in compact orientable 3-manifolds, which are generalizations of those of bridge decompositions and thin positions. We show that either a thin multiple Heegaard splitting of 1-submanifold $T$ is also a Heegaard splitting with minimal complexity or the exterior of $T$ contains an essential surface with meridional boundary other than the boundary parallel annulus.
\end{abstract}

\section{Introduction.}

The notion of thin position for knots in $S^{3}$ is introduced by D. Gabai in $[\mathbf{G}]$, and played important roles in solutions of the property $R$ conjecture by Gabai and the knot complement conjecture by C.McA. Gordon and J. Luecke $[\mathbf{G}-\mathbf{L}]$. A. Thompson showed in $[\mathbf{T}]$ that if an exterior of a knot $K$ does not contain an incompressible planar surface with meridional boundary other than the boundary parallel annulus, then every thin position of $K$ has a level sphere which gives a bridge decomposition of $K$ realizing the bridge number of $K$. M. Scharlemann and Thompson defined thin positions for 3manifolds in $[\mathbf{S}-\mathbf{T}]$, and obtained several results on incompressible surfaces. Recently J. Schultens and K. Morimoto apply results in [S-T] successfully to a problem of tunnel numbers of knots [Sl], [M-Sl]. See also [S-S].

We generalize the main results in $[\mathbf{T}]$ and $[\mathbf{S}-\mathbf{T}]$ and Theorem 3.1 in $[\mathbf{C}-\mathbf{G}]$ in this paper.

The bridge decomposition of a link in the 3 -sphere $S^{3}$ is introduced by $\mathrm{H}$. Schubert [Sb] and generalized by K. Morimoto and M. Sakuma [M-Sa] for a link in a closed orientable 3-manifold. Many researches on such decompositions have appeared by now. See $[\mathbf{D}],[\mathbf{H o}],[\mathbf{K}],[\mathbf{K}-\mathbf{S}],[\mathbf{M}],[\mathbf{M}-\mathbf{S}-\mathbf{Y}]$, [S-Ko], [S-Ki], [H-S1], [H-S2], [H-S3], [Hy1], [Hy2], [Hy3] and [Hy4]. Here we generalize it for a 1-submanifold properly imbedded in a compact orientable 3-manifold possibly with boundary.

Let $I=[0,1]$ an interval, $F$ a disjoint union of closed orientable surfaces. A compression body $C$ is a connected orientable 3-manifold obtained from a ball $B$ or $F \times I$ by attaching some number, perhaps 0 of 1-handles on $\partial B$ or $F \times\{1\}$. Let $\partial_{-} C$ denote $F \times\{0\}$ and $\partial_{+} C=\partial C-\partial_{-} C$. In usual definitions 
$\partial_{-} C$ has no 2 -sphere component, but in this paper $\partial_{-} C$ may have 2 -sphere components. A compression body $C$ is called a handlebody if $\partial_{-} C=\emptyset$.

A set of arcs $\left\{t_{1}, \ldots, t_{n}\right\}$ properly imbedded in a compression body $C$ is trivial if there is a homeomorphism $C \cong Y \cup V$ (where $Y$ is a ball or homeomorphic to $\partial_{-} C \times I$ and $V$ is a disjoint union of 1-handles) such that each arc $t_{i}$ satisfies one of the following conditions.

(1) $t_{i}$ is vertical, i.e., $t_{i}=$ (a point) $\times I \subset \partial_{-} C \times I=Y$, and $t_{i} \cap V=\emptyset$.

(2) $t_{i}$ is $\partial_{+}$-parallel, i.e., there is a disc $D \subset C$ such that $t_{i} \subset \partial D, D \cap \partial C=$ $\operatorname{cl}\left(\partial D-t_{i}\right) \subset \partial_{+} C$ and that $D \cap t_{j}=\emptyset$ for $j \neq i$.

We call the disc $D$ in condition (2) above a cancelling disc of $t_{i}$. A standard cut and paste argument allows us to take mutually disjoint cancelling discs of the $\partial_{+}$-parallel arcs.

It is well known that every compact connected orientable 3-manifold $M$ has a Heegaard splitting $H$, i.e., $M=C_{1} \cup_{H} C_{2}$, where $C_{1}$ and $C_{2}$ are compression bodies and $H=\partial_{+} C_{1}=\partial_{+} C_{2}$. Let $T$ be a properly imbedded 1-manifold in $M$. The Heegaard splitting $H$ of $M$ is a Heegaard splitting of $(M, T)$ if $H$ is transverse to $T$ and $T_{i}=T \cap C_{i}$ is a union of a trivial set of arcs in $C_{i}$ for $i=1$ and 2. Every pair $(M, T)$ as above has a Heegaard splitting (Lemma 2.1).

In general, let $X$ be a compact orientable 3-manifold, and $T$ a 1-manifold properly imbedded in $X$. Let $F$ be a compact (possibly disconnected) orientable 2-manifold properly imbedded in $X$ transversely to $T$. Then $F$ is said to be $T$-compressible if there is a disc $D$ such that $D \cap F=\partial D, D \cap T=\emptyset$ and $\partial D$ is essential in $F-T$, that is, $\partial D$ does not bound a disc in $F-T$. We call such a disc $D$ a $T$-compressing disc of $F$. If $F$ is not $T$-compressible, then it is $T$-incompressible. Let $F_{0}$ and $F_{1}$ be disjoint closed orientable surfaces imbedded in $X$ transversely to $T$. These surfaces are $T$-parallel if they cobound a 3-mamifold homeomorphic to $F_{0} \times I$ possibly intersecting $T$ in vertical arcs, where $F_{0}=F_{0} \times\{0\}$ and $F_{1}=F_{0} \times\{1\}$.

A Heegaard splitting $(M, T)=\left(C_{1}, T_{1}\right) \cup_{H}\left(C_{2}, T_{2}\right)$ is said to be weakly $T$ reducible if there is a $T_{i}$-compressing disc $D_{i} \subset C_{i}$ of $H$ for $i=1$ and 2 such that $\partial D_{1} \cap \partial D_{2}=\emptyset$. Otherwise $H$ is strongly T-irreducible. The splitting $H$ is $T$-reducible if we can take the discs so that $\partial D_{1}=\partial D_{2}$. Otherwise $H$ is $T$-irreducible.

A Heegaard splitting $(M, T)=\left(C_{1}, T_{1}\right) \cup_{H}\left(C_{2}, T_{2}\right)$ is said to be stabilized if there is a properly imbedded disc $D_{i}$ disjoint from $T_{i}$ in $C_{i}$ for $i=1$ and 2 such that $\partial D_{1}$ and $\partial D_{2}$ intersect transversely at a single point in $H$. In this situation, if we performing a $T_{i}$-compressing operation on the splitting surface $H$ along the disc $D_{i}$, then we obtain a new Heegaard splitting surface of $(M, T)$ for $i=1$ and 2 .

Remark. A Heegaard splitting $(M, T)=\left(C_{1}, T_{1}\right) \cup_{H}\left(C_{2}, T_{2}\right)$ is said to be meridionally stabilized if there is a properly imbedded disc $D_{1}$ intersecting $T_{1}$ 
transversely in a single point in $C_{1}$ and if there is a properly imbedded disc $D_{2}$ disjoint from $T_{2}$ in $C_{2}$ such that $\partial D_{1}$ and $\partial D_{2}$ intersect transversely at a single point in $H$. In this situation, if we perform a compressing operation on the splitting surface $H$ along the disc $D_{1}$, then we obtain a new Heegaard splitting surface of $(M, T)$. But we do not use these definition and fact in this paper.

A Heegaard splitting $(M, T)=\left(C_{1}, T_{1}\right) \cup_{H}\left(C_{2}, T_{2}\right)$ is said to be cancellable if there is a cancelling disc $D_{i}$ of an arc $t_{i} \subset T_{i}$ for $i=1$ and 2 such that $\emptyset \neq\left(\partial D_{1} \cap \partial D_{2}\right) \subset(T \cap H)$. In this situation, if $\partial D_{1} \cap \partial D_{2}$ consists of a single point of $T \cap H$, then we can isotope the arc $t_{i}$ along the disc $D_{i}$, to obtain a new Heegaard splitting of $(M, T)$ for $i=1$ and 2 .

A Heegaard splitting $H$ of $(M, T)$ is said to be netted if there is a $T$ compressing disc $D$ of $H$ such that a surgery on $H$ along $D$ yields two surfaces, one of which is $T$-parallel to a component of $\partial M$ and the other is another Heegaard splitting of $(M, T)$.

More generally, a disjoint union $\mathcal{H}$ of closed orientable surfaces imbedded in int $M$ transversely to $T$ is a multiple Heegaard splitting of $(M, T)$ if:

(1) The closures of all components of $M-\mathcal{H}$ are compression bodies $C_{1}, \ldots, C_{n}$

(2) $\partial_{+} C_{i}$ is attached to some $\partial_{+} C_{j}(i \neq j)$ for $i=1, \ldots, n$,

(3) a component of $\partial_{-} C_{i}$ is either attached to some component of $\partial_{-} C_{j}$ (possibly $j=i$ ) or contained in $\partial M$ for $i=1, \ldots, n$, and

(4) $T \cap C_{i}$ is a union of a trivial set of $\operatorname{arcs}$ in $C_{i}$ for $i=1, \ldots, n$.

A component $H$ of $\mathcal{H}$ is said to be positive if $H=\partial_{+} C_{i}$ for some $1 \leq i \leq n$. A component $H$ of $\mathcal{H}$ is said to be negative if $H \subset \partial_{-} C_{i}$ for some $1 \leq i \leq n$. Let $\mathcal{H}_{+}$and $\mathcal{H}_{-}$denote the disjoint union of all positive surfaces of $\mathcal{H}$ and the disjoint union of all negative surfaces of $\mathcal{H}$ respectively. Note that $\mathcal{H}$ may contain a surface which is non-separating in $M$.

Let $W_{i j}=C_{i} \cup C_{j}$ be a component of the 3-manifold obtained by cutting $M$ along $\mathcal{H}_{-}$, where $\partial_{+} C_{i}=\partial_{+} C_{j}=H_{i j} \subset \mathcal{H}_{+}$. Let $T_{i j}=T \cap W_{i j}$. We say that the splitting $\mathcal{H}$ is slim if the splitting $H_{i j}$ of $\left(W_{i j}, T_{i j}\right)$ is strongly $T$-irreducible for all $W_{i j}$, and if any proper subset of $\mathcal{H}$ is not a multiple Heegaard splitting of $(M, T)$.

We will define a width of a multiple Heegaard splitting of $(M, T)$. Let $S$ be a closed connected orientable surface imbedded in $M$ transversely to $T$. The complexity of $S$ is the ordered pair $c(S)=$ (genus $(S),|S \cap T|)$. We order complexities lexicographically. The width of a multiple Heegaard splitting $\mathcal{H}$ is the multi-set of pairs $w(\mathcal{H})=\{c(S) \mid S$ is a component of $\left.\mathcal{H}_{+}\right\}$, where this "multi-set" may contain the same ordered pairs redundantly. For example, $w(\mathcal{H})=\{(5,7),(3,4),(3,4),(2,1),(2,0)\}$ or $w\left(\mathcal{H}^{\prime}\right)=$ $\{(5,7),(3,4),(2,8),(2,0),(1,7),(1,7)\}$. We order finite multi-sets of pairs 
as follows: Arrange ordered pairs in each multi-set in monotonically nonincreasing order, then compare the ordered multi-sets lexicographically. In the above example, we have $w(\mathcal{H})>w\left(\mathcal{H}^{\prime}\right)$. These definitions of width and its ordering are in imitation of $[\mathbf{S}-\mathbf{T}]$. Define the width $w(M, T)$ to be the minimal width over all multiple Heegaard splittings of $(M, T)$ with respect to the above ordering. We say $(M, T)$ is in thin position if the width of the given multiple Heegaard splitting $\mathcal{H}$ realizes the width $w(M, T)$. We say also that the multiple Heegaard splitting $\mathcal{H}$ is thin. We see later a thin multiple Heegaard splitting is slim in Lemma 2.3.

Remark. If we define the complexity $c(S)=-\chi(S-\operatorname{int} N(T))$, then we obtain another definition of thin position. All results in this paper also hold for this definition.

In general, let $X$ be a compact orientable 3-manifold, $T$ a 1-manifold properly imbedded in $X$, and $F$ a closed orientable 2-manifold imbedded transversely to $T$ in $X$. Let $\tilde{X}$ be the 3 -manifold obtained from $X$ by capping off all the spherical boundary components disjoint from $T$ with balls. An imbedded disc $Q$ is said to be a thinning disc of $F$ if $T \cap Q=T \cap \partial Q=\alpha$ is an arc and $Q \cap F$ contains the $\operatorname{arc} \operatorname{cl}(\partial Q-\alpha)=\beta$ as a connected component. Note that int $Q$ may intersect $F$. A closed 2-manifold $F$ is $T$-essential if (1) $F$ is $T$-incompressible, (2) $F$ has no thinning disc and (3) no component of $F$ is $T$-parallel to a component of $\partial X$ in $\tilde{X}$ and (4) no sphere component of $F$ bounds a ball disjoint from $T$ in $\tilde{X}$.

The surface $F \cap(X-\operatorname{int} N(T))$ is incompressible and $\partial$-incompressible in $X$-int $N(T)$ when $\partial X=\emptyset$ and $F$ is $T$-essential.

Theorem 1.1. Let $M$ be a compact connected orientable 3-manifold, and $T$ a 1-manifold properly imbedded in $M$. Suppose $\mathcal{H}$ is a slim multiple Heegaard splitting of $(M, T)$. Then $\mathcal{H}_{-}$is $T$-essential in $(M, T)$. In addition a component of $\partial M$ is $T$-incompressible in $(M, T)$ if it is not $T$-parallel to any component of $\mathcal{H}_{+}$in $\tilde{M}$, where $\tilde{M}$ is the 3-manifold obtained from $M$ by capping off all the spherical boundary components disjoint from $T$ with balls.

Note that if $\mathcal{H}_{-}=\emptyset$, then it is a non-multiple Heegaard splitting, that is, $\mathcal{H}$ consists of only one component of positive surface $H$, and hence $H$ is a Heegaard splitting of $(M, T)$. When $M=S^{3}$ and surfaces of $\mathcal{H}$ are spheres, Theorem 1.1 is similar to Theorem 1 in $[\mathbf{T}]$. In $[\mathbf{H}-\mathbf{K}]$, D.J. Heath and T. Kobayashi improved Theorem 1 in $[\mathbf{T}]$. When $T=\emptyset$, it is similar to Rules 1 and 5 in $[\mathbf{S}-\mathbf{T}]$. This result was independently obtained by C. Feist in $[\mathbf{F}]$.

Theorem 1.2. Let $M$ be a compact connected orientable 3-manifold, and $T$ a 1-manifold properly imbedded in $M$. Suppose $H$ is a T-irreducible and weakly T-reducible Heegaard splitting of $(M, T)$. Then there is an untelescoping operation (defined in Lemma 2.3) which yields a multiple Heegaard 
splitting $\mathcal{H}$ such that $w(\mathcal{H})<w(\{H\})$, and $\mathcal{H}_{-}$contains a non-empty $T$ incompressible surface $F$ which is not a sphere disjoint from $T$.

When $H$ is not cancellable, we can take $F$ so that $F$ is not a sphere which bounds in $\tilde{M}$ a 3-ball intersecting $T$ in a trivial arc and so that $F$ is not a torus which bounds in $\tilde{M}$ a solid torus intersecting $T$ in a core loop of $V$, where $\tilde{M}$ is the 3-manifold obtained from $M$ by capping off all the spherical boundary components disjoint from $T$ with balls. Moreover, when $H$ is not cancellable and not netted, we can take $F$ so that $F$ is not a surface which is T-parallel to a component of $\partial \tilde{M}$ in $\tilde{M}$.

A.J. Casson and C.McA. Gordon proved the above theorem in the case where $T=\emptyset$ in [C-G, Theorem 3.1]. The untelescoping operation is introduced in the proof of $[\mathbf{C - G}$, Theorem 3.1] and formulated in [S-T]. See also [L-M, Theorem 1.3].

Remark. Let $F$ be a closed orientable surface. Then $(F \times I$, vertical $\operatorname{arcs} T)$ has a Heegaard splitting $H$ which is $T$-parallel to the boundary components $F \times\{0\}$ and $F \times\{1\}$. Let $H^{\prime}$ be a Heegaard splitting of $(F \times I, T)$ such that a cancelling operation on $H^{\prime}$ yields $H$. Then $H^{\prime}$ is cancellable, but is not weakly $T$-reducible.

Hence, one might think that Theorem 1.2 needs cancelling operations besides untelescoping operations. However, a cancelling operation does not change the negative surfaces $\mathcal{H}_{-}$, and keeps $T$ - $\partial$-incompressibility. Thus we do not need cancelling operations in Theorem 1.2.

In the proofs of Theorems 1.1 and 1.2, we need the next Theorem 1.3.

Let $X$ be a 3 -manifold, and $T$ a 1-manifold properly imbedded in $X$. Let $F$ be a 2-manifold properly imbedded in $X$ transversely to $T$, and $D$ a disc imbedded in $X$ so that $D \cap F=\partial D$ and so that $D \cap T=\emptyset$. A 2-surgery on $F$ along $D$ is such an operation as below. Take a tubular neighbourhood $N(D) \cong D \times[0,1]$ of $D$ so that $N(D) \cap F=\partial D \times[0,1]$ and so that $N(D) \cap T=\emptyset$. Then replace the annulus $\partial D \times[0,1]$ on $F$ with the two discs $D \times\{0\} \cup D \times\{1\}$. We call this operation a $T$-compressing on $F$ along $D$ if $D$ is a $T$-compressing disc of $F$.

Let $M$ be a 3-manifold, and $T$ a 1-manifold properly imbedded in $M$. The pair $(M, T)$ is split if the complement $C(T)=M-T$ contains an essential sphere $S$, that is, $S$ does not bound a ball in $C(T)$. This sphere $S$ is called a splitting sphere.

Theorem 1.3. Let $M$ be a compact connected orientable 3-manifold, and $T$ a 1-manifold properly imbedded in $M$. Let $H$ be a Heegaard splitting of $(M, T)$, and $S$ a disjoint union of splitting spheres in $(M, T)$ and $T$ compressing discs of $\partial M$. Then there is a set of a disjoint union of splitting spheres and T-compressing discs $S^{\prime}$ such that:

(1) $S^{\prime}$ is obtained from $S$ by 2-surgeries and isotopy in $(M, T)$, 
(2) each sphere of $S^{\prime}$ intersects $H$ in at most one simple closed curve, and

(3) each disc of $S^{\prime}$ intersects $H$ in precisely one simple closed curve essential on $H-T$.

When $\partial M=\emptyset$ and $T=\emptyset$, this is a theorem of $\mathrm{W}$. Haken $[\mathbf{H k}$, Theorem in Section 7]. Section 7 of $[\mathbf{H k}]$ is readable independently without reading the other sections. W. Jaco gave a slightly easier proof of Haken's theorem [Ja, Theorem II.7]. See also [Jo, Proposition 3.2] and [O, Theorem 1]. B.F. Bonahon and J.P. Otal showed the above theorem when $T=\emptyset[\mathbf{B}-\mathbf{O}$, Proposition 8]. See also [C-G, Lemma 1.1]. H. Doll proved the above theorem in the case where $\partial M=\emptyset[\mathbf{D}$, Theorem 1.6]. The proof of Theorem 1.3 is similar to that of [Hk, Theorem in Section 7] and [Ja, Theorem II.7].

The next is a corollary of Theorem 1.3. This is a generalization of [Lemma 1.1(iii), C-G].

Corollary 1.4. Let $H$ be a strongly T-irreducible Heegaard splitting of $(M, T)$. Let $\tilde{M}$ be the 3-manifold obtained by capping off all the spherical boundary components disjoint from $T$ with balls. Let $F$ be a component of $\partial \tilde{M}$. If $H$ is not $T$-parallel to $F$ in $\tilde{M}$, then $F$ is $T$-incompressible.

We prove Theorem 1.3 and Corollary 1.4 first in Sections 3 and 4, Theorem 1.1 in Section 5 and Theorem 1.2 in Section 6. Though the proofs of Theorems 1.1 and 1.2 use Theorem 1.3 and Corollary 1.4, they are readable without reading Sections 3 and 4 .

\section{Preliminaries.}

A spine $X$ of a compression body $C$ is an imbedded (possibly disconnected) 1-complex such that $X$ intersects $\partial_{-} C$ in vertices, $X \cap \partial_{+} C=\emptyset$ and $\operatorname{cl}(C-$ $N(X))$ is homeomorphic to $\partial_{+} C \times I$. $X$ is allowed to be a 0 -cell when $C$ is a ball.

Lemma 2.1. Let $M$ be a compact connected orientable 3-manifold. Suppose that a surface $H$ gives a Heegaard splitting $M=C_{1} \cup_{H} C_{2}$. Let $T$ be a 1manifold properly imbedded in $M$. Then we can isotope $T$ in $M$ so that $H$ is a Heegaard splitting of $(M, T)$.

Proof. Let $X_{i}$ be a spine of the compression body $C_{i}$ for $i=1$ and 2 . We can isotope $T$ to be disjoint from small neighbourhoods $N\left(X_{1}\right), N\left(X_{2}\right)$ of the spines $X_{1}, X_{2}$. Then $M-\operatorname{int}\left(N\left(X_{1}\right) \cup N\left(X_{2}\right)\right)$ is homeomorphic to $H \times I$ where $H=H \times\{1 / 2\}$ and $H \times\{0\} \subset C_{1}$. Let $\pi$ be the projection $H \times I \rightarrow H$. We can take this product structure so that the singular set of $\pi(T)$ consists of double points away from $\partial T$. Let $t$ be a component of $T$, and $\partial_{1} t, \partial_{2} t$ endpoints of $t$ if $t$ is an arc. We say that $\partial_{i} t$ is lower and upper if it is in $\partial_{-} C_{1}$ and $\partial_{-} C_{2}$ respectively. Let $S$ be the set consisting of $\partial_{1} t, \partial_{2} t$ and singular points on $t$. We take regular points of $t$, one between 
every pair of adjacent points of $S$. We take regular points of $t$, one more between adjacent points of $S$ if the both points are upper, or both are lower. In addition we take two regular points of $t$ if $t$ is a circle without singular points. Then we can isotope $T$ so that $T \cap H$ consists of the above regular points and $T \cap C_{i}$ is trivial in $C_{i}$ for $i=1$ and 2 .

In general, a properly imbedded arc $\alpha$ in a 2-manifold $F$ is inessential if there exists an arc $\beta \subset \partial F$ such that $\alpha \cup \beta$ forms a loop bounding a disc in $F$. Otherwise, $\alpha$ is essential.

Let $X$ be a compact orientable 3 -manifold, and $T$ a 1-manifold properly imbedded in $X$. Let $F$ be a compact orientable 2-manifold properly imbedded in $X$ so that it is transverse to $T$. Then $F$ is said to be $T$ - $\partial$-compressible if there is a disc $D$ such that $D \cap T=\emptyset, D \cap F=\partial D \cap F=\alpha$ is an essential arc in $F-T$ and $D \cap \partial X=\operatorname{cl}(\partial D-\alpha)$. We call this disc $D$ a $T$ - $\partial$-compressing disc of $F$. If $F$ is not $T$ - $\partial$-compressible, then it is $T$ - $\partial$-incompressible.

Let $(C, T)$ be a pair of a compression body $C$ and trivial $\operatorname{arcs} T$ in $C$. Let $\mathcal{D}$ be a disjoint union of (1)discs disjoint from $T$ and with their boundaries in $\partial_{+} C$ and (2)cancelling discs, one for each $\partial_{+}$-parallel arc of $T$. This union $\mathcal{D}$ of discs is a complete disc system of $(C, T)$ if $\mathcal{D}$ cuts $(C, T)$ into a manifold which is homeomorphic to disjoint union of $\left(\partial_{-} C \times I\right.$, vertical arcs $)$ and some number, perhaps 0 of balls possibly with arcs of $T$ in its boundary.

We can take a complete disc system of $(C, T)$ as follows. First we take a disjoint union of cancelling discs $\mathcal{D}^{\prime}$, one for each $\partial_{+}$-parallel arc of $T$. There is a homeomorphism $C \cong Y \cup V$, where $Y$ is a 3 -ball or $\partial_{-} C \times I$ and $V$ is a disjoint union of 1-handles, such that vertical arcs of $T$ are (points) $\times I \subset \partial_{-} C \times I$, and are disjoint from $V$. We can take cocore discs $\mathcal{D}^{\prime \prime}$ of $V$. We isotope $\mathcal{D}^{\prime} \cup T$ so that $\partial_{+}$-parallel arcs of $T$ are very close to the arcs $\partial \mathcal{D}^{\prime} \cap \partial_{+} C$ and so that $\mathcal{D}^{\prime} \cap \mathcal{D}^{\prime \prime}$ consists of arcs connecting $T$ and $\partial \mathcal{D}^{\prime} \cup \partial_{+} C$ on $\mathcal{D}^{\prime}$. We can isotope $\mathcal{D}^{\prime \prime}$ in $C$ to be disjoint from $\mathcal{D}^{\prime} \cap T$. Then $\mathcal{D}=\mathcal{D}^{\prime} \cup \mathcal{D}^{\prime \prime}$ is a complete disc system of $(C, T)$.

Let $(C, T)$ be as above. We cap off each sphere component of $\partial_{-} C$ with a 3-ball if it is disjoint from $T$, to obtain a new compression body denoted by $\tilde{C}$ throughout this paper.

Lemma 2.2. Let $(C, T)$ be a pair of a compression body $C$ and trivial arcs $T$ in $C$. Let $S$ be a $T$-incompressible and $T$ - $\partial$-incompressible 2 -manifold in $(C, T)$. Then there is a complete disc system $\mathcal{D}$ of $(\tilde{C}, T)$ such that $\mathcal{D}$ is properly imbedded in $C$ and $\mathcal{D} \cap S$ consists of two types of arcs as below.

(1) An intersection arc $\alpha$ of a cancelling disc of $\mathcal{D}$ and a sphere intersecting $T$ in two points. Both endpoints of $\alpha$ are in $T$.

(2) An intersection arc $\beta$ of a cancelling disc of $\mathcal{D}$ and a disc intersecting $T$ in one point. One endpoint of $\beta$ is in $T$ and the other is in $\partial_{+} C$. 


\section{Proof.}

Step 1. Let $Z=\operatorname{cl}(\tilde{C}-C)$ the disjoint union of balls. Let $\mathcal{D}$ be a complete disc system of $(\tilde{C}, T)$ which is disjoint from $Z$. We can isotope $S$ slightly so that $S$ is transverse to $\mathcal{D}$. Suppose that $S \cap \mathcal{D}$ contains simple closed curves, then there is an innermost one on $\mathcal{D}$. This closed curve bounds an innermost disc $D$ whose interior is disjoint from $S$. Since $S$ is $T$-incompressible, there is a disc $D^{\prime}$ on $S$ such that $\partial D^{\prime}=\partial D$ and $D^{\prime} \cap T=\emptyset$. Let $D^{\prime \prime}$ be an innermost disc bounded by a loop of $S \cap \mathcal{D}$ on $D^{\prime}$, and $D^{\prime \prime \prime}$ be the disc bounded by $\partial D^{\prime \prime}$ on $\mathcal{D}$. We change $\mathcal{D}$ by removing $D^{\prime \prime \prime}$ and attaching $D^{\prime \prime}$, and a small isotopy of $\mathcal{D}$ decreases the number $|S \cap \mathcal{D}|$. The sphere $D^{\prime \prime} \cup D^{\prime \prime \prime}$ bounds in $\tilde{C}$ a ball which is disjoint from $T$, and hence $\mathcal{D}$ remains to be a complete disc system of $(\tilde{C}, T)$. We repeat this operation until $S \cap \mathcal{D}$ consists of arcs only.

Step 2. Suppose that $S \cap \mathcal{D}$ contains arcs. Let $\alpha$ be an outermost arc of $S \cap \mathcal{D}$ on $\mathcal{D}$, and $D$ the outermost disc, that is, $D \cap S=\alpha$. Suppose first that $(\partial D-\alpha) \subset \partial_{+} C$. Then $\alpha$ is inessential on $S-T$ since $S$ is $T$ - $\partial$ incompressible. Hence there is an $\operatorname{arc} \beta$ of $S \cap \mathcal{D}$ which is inessential and outermost on $S-T$. This arc $\beta$ cuts off a disc $R$ from $S-T$ such that $R \cap \mathcal{D}=\beta$. We perform a surgery on $\mathcal{D}$ along $R$, that is, we replace a small neighbourhood of $\beta$ on $\mathcal{D}$ by two parallel copies of $R$. Then we obtain a new complete disc system of $(\tilde{C}, T)$. (Note that we can retake the product structure $\partial_{-} \tilde{C} \times I$ so that vertical arcs remains vertical.) We repeat this operation until there is no such outermost disc $D$.

Step 3. Suppose secondly that $(\partial D-\alpha) \subset T$. Let $C^{\prime}$ be the 3-manifold obtained from $C$ by cutting along $S$. We take a regular neighbourhood $N(D)$ of $D$ in $C^{\prime}$. Then $D^{\prime}=\operatorname{cl}\left(\partial N(D)-\partial C^{\prime}\right)$ is a disc such that $D^{\prime} \cap S=\partial D^{\prime}$. Since $S$ is $T$-incompressible, there is a disc $D^{\prime \prime}$ on $S-T$ such that $\partial D^{\prime \prime}=\partial D^{\prime}$. Hence the component of $S$ containing $\alpha$ is a sphere $S^{\prime}$ intersecting $T$ in two points. That is, $\alpha$ is of type (1). Note that $S-S^{\prime}$ is $T$-incompressible and $T$ - $\partial$-incompressible. We repeat this operation on $S-S^{\prime}$, to see that we can assume there is no such disc $D$.

Step 4. Similar argument as in Step 3 shows that an arc of $\mathcal{D} \cap S$ is of type (2) if one endpoint is in $T$ and the other is in $\partial_{+} C$.

The next lemma is similar to Rule 3 in $[\mathbf{S}-\mathbf{T}]$, and implies that a thin multiple Heegaard splitting is slim.

Lemma 2.3. Let $\mathcal{H}$ be a thin multiple Heegaard splitting of ( $M$, $T)$. Then no component $H_{i j}$ of $\mathcal{H}_{+}$is a weakly $T$-reducible Heegaard splitting of $\left(W_{i j}, T_{i j}\right)$, where $W_{i j}$ is the component of the 3-manifold obtained by cutting $M$ along $\mathcal{H}_{-}$and containing $H_{i j}$, and $T_{i j}=T \cap W_{i j}$. 
Proof. Suppose, for a contradiction, that some $H_{i j}$ is weakly $T$-reducible. Let $C_{i}, C_{j}$ be the compression bodies obtained by cutting $W_{i j}$ along $H_{i j}$. We will decompose $W_{i j}$ into compression bodies with fewer width. This operation is called untelescoping. Since $H_{i j}$ is weakly $T$-reducible, there is a non-empty disjoint union $\mathcal{D}_{m}$ of $T$-compressing discs of $H_{i j}$ in $\left(C_{m}, T_{m}\right)$ for $m=i$ and $j$ such that $\partial \mathcal{D}_{i} \cap \partial \mathcal{D}_{j}=\emptyset$. Let $C_{1}^{\prime}=\operatorname{cl}\left(C_{i}-N\left(\mathcal{D}_{i}\right)\right)$ and $C_{4}^{\prime}=\operatorname{cl}\left(C_{j}-N\left(\mathcal{D}_{j}\right)\right)$. Then $C_{k}^{\prime}$ is a disjoint union of compression bodies for $k=1$ and 4 such that $\partial_{-} C_{1}^{\prime}=\partial_{-} C_{i}$ and $\partial_{-} C_{4}^{\prime}=\partial_{-} C_{j}$. Note that $T \cap C_{k}^{\prime}$ is trivial in $C_{k}^{\prime}$ for $k=1$ and 4 . (The union of compression bodies $C_{1}^{\prime}$ and $C_{4}^{\prime}$ may have a ball component disjoint from $T$.) We take a sufficiently small collar $N\left(\partial_{+} C_{k}^{\prime}\right)$ of $\partial_{+} C_{k}^{\prime}$ in $C_{k}^{\prime}$ so that $T \cap N\left(\partial_{+} C_{k}^{\prime}\right)$ is a disjoint union of vertical arcs for $k=1$ and 4 . Let $C_{k}=\operatorname{cl}\left(C_{k}^{\prime}-N\left(\partial_{+} C_{k}^{\prime}\right)\right)$ for $k=1$ and $4, C_{2}=N\left(\partial_{+} C_{1}\right) \cup N\left(\mathcal{D}_{j}\right)$ and $C_{3}=N\left(\partial_{+} C_{4}\right) \cup N\left(\mathcal{D}_{i}\right)$. These are disjoint unions of compression bodies such that $\partial_{+} C_{1}=\partial_{+} C_{2}$ and $\partial_{+} C_{3}=\partial_{+} C_{4}$. Then the complexity of $H_{i j}=\partial_{+} C_{i}=\partial_{+} C_{j}$ is larger than that of any component of $\partial_{+} C_{k}$ for $k=1,2,3$ or 4 . Thus we obtain a multiple Heegaard splitting of $\left(W_{i j}, T_{i j}\right)$, hence that of $(M, T)$ with smaller width. This is a contradiction.

Let $(C, T)$ be a pair of a compression body $C$ and trivial arcs $T$ in $C$. An annulus $A$ properly imbedded in $C$ is a vertical annulus, if there is a homeomorphism $C \cong\left(\partial_{-} C \times I\right) \cup V$ (where $V$ is a disjoint union of 1handles) such that:

(1) The vertical arc components of $T$ are vertical in $\partial_{-} C \times I$,

(2) $\partial_{+}$-parallel arc components of $T$ are disjoint from $A$, and

(3) $A=\ell \times I \subset \partial_{-} C \times I$ and $A \cap V=\emptyset$ where $\ell$ is a simple closed curve in $\partial_{-} C$.

The next lemma is a mild generalization of Lemma 9 in [B-O].

Lemma 2.4. Let $(C, T)$ be a pair of a compression body $C$ and trivial arcs $T$ in $C$. Let $S$ be a $T$-incompressible and $T$ - $\partial$-incompressible 2-manifold properly imbedded in $C$ transversely to $T$. Then each component of $S$ is either:

(1) A sphere intersecting $T$ at 0 or 2 points,

(2) a disc intersecting $T$ at most 1 point,

(3) a vertical annulus disjoint from $T$, or

(4) a closed surface $T$-parallel to a component of $\partial_{-} \tilde{C}$ in $(\tilde{C}, T)$.

Proof.

Step 1. We consider the union of surfaces obtained from $S$ deleting all the surfaces of types (1) and (2). We let $S$ denote the resulting 2-manifold for simplicity of notation. It is sufficient to show that each component of $S$ is of type (3) or (4). 
Step 2. Let $\mathcal{D}$ be a complete disc system of $(\tilde{C}, T)$ as in Lemma 2.2. Note that $\mathcal{D} \cap S=\emptyset$ since $S$ does not contain surfaces of types (1), (2). Hence $S$ is disjoint from $\partial_{+}$-parallel $\operatorname{arcs}$ of $T$. The discs $\mathcal{D}$ cuts $\tilde{C}$ into a 3-manifold which is homeomorphic to disjoint union of $\partial_{-} \tilde{C} \times I$ and balls. Then $S$ does not intersect these balls since incompressible surfaces in a ball are spheres and discs.

Step 3. Let $\ell$ be a disjoint union of simple closed curves in $\partial_{-} \tilde{C}$ such that $\ell$ is essential in non-sphere components of $\partial_{-} \tilde{C}$ and $\ell$ decomposes spheres of $\partial_{-} \tilde{C}$ into several discs, tori into annuli and the other components into pairs of pants, that is, spheres with three holes. Let $\mathcal{A}=\ell \times I$ the disjoint union of vertical annuli in $\tilde{C}$. We can take $\ell$ and the product structure $\partial_{-} \tilde{C} \times I$ so that $\mathcal{A}$ is disjoint from $\mathcal{D} \cup Z$ and so that $\mathcal{A}$ contains all vertical $\operatorname{arcs}$ of $T$. In particular, an annulus of $\mathcal{A}$ must contain a vertical arc if it is incident to a sphere component of $\partial_{-} \tilde{C}$. (Note that the closure of every component of $\mathcal{A}-T$ is " $T$-incompressible" and " $T$ - $\partial$-incompressible".) We can deform $\mathcal{A}$ so that $S \cap \mathcal{A}$ does not contain an inessential loop on $\mathcal{A}-T$ as in Step 1 in the proof of Lemma 2.2, and so that $S \cap \mathcal{A}$ does not contain an inessential arc on $\mathcal{A}-T$ as in Step 2 in the proof of Lemma 2.2. Similar arguments as in Steps 3, 4 in the proof of Lemma 2.2 show that every loop of $S \cap \mathcal{A}$ is essential on $\mathcal{A}$ and does not intersect a vertical arc of $T$ more than once, and that any arc of $S \cap \mathcal{A}$ does not intersect $T$. Then the $\operatorname{arcs}$ of $S \cap \mathcal{A}$ are vertical. We can assume that $|S \cap \mathcal{A}|$ is minimal up to isotopy of $S$ in $(C, T)$ and over all choices of $\mathcal{A}$.

Step 4. For each annulus (or pair of pants) $P$ in $\partial_{-} \tilde{C}$, we take two arcs (or three arcs) $\gamma$ properly imbedded in $P$ such that $\partial \gamma$ is disjoint from $T$ and $\gamma$ cuts $P$ into two square discs (or two hexagonal discs). Let $\mathcal{B}=\gamma \times I$ the disjoint union of vertical discs. We can take $\gamma$ and the product structure $\partial_{-} \tilde{C} \times I$ so that $\mathcal{B}$ is disjoint from $Z \cup \mathcal{D} \cup T$ and $\partial \gamma \cap S=\emptyset$. We can deform $\mathcal{B}$ so that $S \cap \mathcal{B}$ consists of arcs only as in Step 1 in the proof of Lemma 2.2. (Let $\ell$ be an innermost loop of $S \cap \mathcal{B}$ on $\mathcal{B}$. Then there is a disc $D^{\prime}$ in $S$ such that $\partial D^{\prime}=\ell$ and $D^{\prime} \cap T=\emptyset$. Note that $D^{\prime}$ is disjoint from $\mathcal{A}$.) We can deform $\mathcal{B}$ so that $S \cap \mathcal{B}$ does not contain an inessential arc whose both endpoints are in $\mathcal{B} \cap \partial_{+} \tilde{C}$ or $\mathcal{B} \cap \partial_{-} \tilde{C}$ as in Step 2 in the proof of Lemma 2.2. Suppose that there is an arc $\alpha$ of $S \cap \mathcal{B}$ such that $\partial \alpha$ is contained in a component of $(\partial \gamma) \times I$. We take $\alpha$ to be outermost on $\mathcal{B}$, and isotope $S$ along the outermost disc. Then two essential loops on $\partial P \times I$ are deformed into an inessential loop on $\mathcal{A}$, and we can decrease the number $|S \cap \mathcal{A}|$, which is a contradiction. Suppose that there is an $\operatorname{arc} \beta$ of $S \cap \mathcal{B}$ such that one of the poins $\partial \beta$ is contained in $(\partial \gamma) \times I$ and the other is contained in $\gamma \times(\partial I)$. We take $\beta$ to be outermost on $\mathcal{B}$, and isotope $S$ along the outermost disc. Then an essential loop on $\partial P \times I$ is deformed into an inessential arc on an annulus $A$ of $\mathcal{A}$. If $A$ is disjoint from $T$, then as in Step 2 in the proof of 
Lemma 2.2 we can decrease the number $|S \cap \mathcal{A}|$, which is a contradiction. If $A$ contains a vertical arc of $T$, then as in Step 4 in the proof of Lemma 2.2 we find a disc component of $S$ intersecting $T$ in a single point, which contradicts our assumption in Step 1 in this proof. Hence the $\operatorname{arcs}$ of $S \cap \mathcal{B}$ consists of vertical arcs and arcs connecting two components of $(\partial \gamma) \times I$.

Step 5. We cut $\tilde{C}$ along the discs of $\mathcal{D}$, and obtain a 3 -manifold homeomorphic to $\partial_{-} \tilde{C} \times I$. We cut it further along the surfaces $\mathcal{A} \cup \mathcal{B}$. For each (possibly square or hexagonal) disc $G$ in $\partial_{-} \tilde{C}, X=G \times I$ is homeomorphic to a ball. Since $S$ is $T$-incompressible, each component of $S \cap(X-\operatorname{int} Z)$ is a disc intersecting $\partial G \times I$.

We show that the boundary of each disc of $S \cap X$ is contained in $\partial G \times I$ or meets $\partial G \times I$ in precisely 2 vertical arcs. Suppose not. Then there is a disc component $Q \subset S \cap X$ meeting $\partial G \times I$ in at least 4 vertical arcs. It follows that $\partial Q$ meets each of $G$ and $\partial X \cap \partial_{+} C$ in at least two subarcs. Cutting the sphere $\partial X$ along $\partial Q$, we obtain two discs, with each of which $Q$ cobounds a ball in $X$. Hence there is a disc $D$ in $X$ such that $D \cap Z=\emptyset$, $D \cap Q=\partial D \cap Q=\alpha$ is an arc and the complementary arc $\beta=\operatorname{cl}(\partial D-\alpha) \subset G$ connects two distinct components of $\partial Q \cap G$. We take $Q$ and $D$ so that $|D \cap S|$ is minimal over all such discs.

Suppose, for a contradiction, that $S$ meets the interior of $D$. We can easily see that the intersection of $D$ and the discs $S \cap X$ contains no closed curves. Let $\rho$ be an outermost arc of $S \cap D$, i.e., $\rho$ and a subarc of $\partial D$ cobounds a subdisc $D^{\prime}$ of $D$ such that $D^{\prime}-\rho$ is disjoint from $S$. Let $R$ be the component of $S \cap X$ which contains $\rho$.

Suppose first that $\partial \rho$ is contained in the same component $\mu$ of $\partial R \cap G$. Let $R^{\prime}$ be the subdisc of $R$ cobounded by the arc $\rho$ and a subarc of $\mu$. We take an arc $\rho^{\prime}$ of $R^{\prime} \cap D$ to be outermost on $R^{\prime}$. Let $R^{\prime \prime}$ be the outermost disc cut off from $R^{\prime}$ by $\rho^{\prime}$. Then we surger $D$ along $R^{\prime \prime}$, and obtain two discs. One of them, say $D^{\prime}$, contains $\alpha$, and $\left|D^{\prime} \cap S\right|<|D \cap S|$, which is a contradiction.

Suppose secondly that $\partial \rho$ is contained in distinct components of $\partial R \cap G$. Then $\partial R$ meets $\partial G \times I$ in at least 4 vertical arcs. This contradicts the minimality of the number $|D \cap S|$. Hence we obtain $D \cap S=\alpha$.

Since each component of $Q-\alpha$ contains components of $\partial S \cap \partial X \cap \partial_{+} C$, $\alpha$ is essential in $S$. Hence $D$ is a $T$ - $\partial$-compressing disc of $S$, which is a contradiction. Thus we have shown that the intersection of $S$ and each $G \times I$ consists of discs whose boundary is contained in $\partial G \times I$ or meets $\partial G \times I$ in precisely 2 vertical arcs.

Step 6 . The discs of the former type together form surfaces $T$-parallel to components of $\partial_{-} \tilde{C}$ in $(\tilde{C}, T)$. The discs of the latter type together form vertical annuli disjoint from $T$ in $(C, T)$. 


\section{Hierarchy of planar surfaces.}

The next lemma is essentially due to Jaco [Ja, Lemma II.9]. A weak hierarchy for a compact orientable 2-manifold $F$ is a sequence of pairs $\left(F_{0}, \alpha_{0}\right), \ldots$, $\left(F_{n}, \alpha_{n}\right)$ where $F_{0}=F, \alpha_{i}$ is an essential arc or simple closed curve in $F_{i}$, $F_{i+1}$ is obtained from $F_{i}$ by cutting along $\alpha_{i}$, and $F_{n+1}$ satisfies the following conditions.

(1) Each component of $F_{n+1}$ is a disc or an annulus at least one boundary component of which is a component of $\partial F_{0}$.

(2) Each non-annular component of $F_{0}$ has at most one boundary component which survives in $\partial F_{n+1}$.

Lemma 3.1. Let $F$ be a connected planar surface. Assume that $F$ has $b$ boundary components. Let $\left(F_{0}, \alpha_{0}\right), \ldots,\left(F_{n}, \alpha_{n}\right)$ be any weak hierarchy for $F$ with each $\alpha_{i}$ an arc. Let $d$ be the number of components of $\partial F_{n+1}$. Then:

(1) $d \leq b-1$ if $b \geq 2$ and if $F_{n+1}$ does not contain an annulus component,

(2) $d \leq b$ if $b \geq 2$ and if $F_{n+1}$ contains an annulus component. When $b \geq 3$ and $d=b, F_{n+1}$ contains a disc component.

Proof. (1) is a lemma of W. Jaco [Ja, Lemma II.8], and we omit the proof. The proof of (2) is very similar to that of (1) by Jaco, but we include it here for convenience of readers.

The proof of $d \leq b$ is via induction on $b$. When $b=2, F_{0}=F_{n+1}$ and clearly $d=b=2$. Suppose that $F$ has $b$ boundary components where $b \geq 3$, and that $d \leq b$ is true for all connected planar surfaces having fewer than $b$ boundary components. There are two cases.

Case I. $\alpha_{0}$ does not separate $F$.

Set $b_{1}$ equal to the number of boundary components of $F_{1}$. Since $\alpha_{0}$ does not separate $F$ and $F$ is planar, distinct end points of $\alpha_{0}$ are in distinct components of $\partial F$; and it follows that $b_{1}=b-1$. Hence, by induction, $d \leq b_{1}=b-1$.

Case II. $\alpha_{0}$ separates $F$.

Let $F_{1}^{\prime}$ and $F_{1}^{\prime \prime}$ denote the components of $F_{1}$, where $F_{1}^{\prime}$ contains the boundary component of $\partial F$ which survives in $F_{n+1}$. Set $b_{1}, b_{1}^{\prime}$ and $b_{1}^{\prime \prime}$ equal to the number of boundary components of $F_{1}, F_{1}^{\prime}$ and $F_{1}^{\prime \prime}$, respectively. Set $d^{\prime}$ and $d^{\prime \prime}$ equal to the number of boundary components of $F_{n+1}$ which are derived from $\partial F_{1}^{\prime}$ and $\partial F_{1}^{\prime \prime}$, respectively. Since $\alpha_{0}$ separates $F$, distinct end points of $\alpha_{0}$ are in the same component of $\partial F$; and it follows that $b_{1}=b+1$. Both $b_{1}^{\prime} \geq 2$ and $b_{1}^{\prime \prime} \geq 2$ because $\alpha_{0}$ is essential. In addition, $d^{\prime \prime} \leq b_{1}^{\prime \prime}-1$ by (1), and $d^{\prime} \leq b_{1}^{\prime}$ by induction. Hence $d=d^{\prime}+d^{\prime \prime} \leq b_{1}^{\prime}+\left(b_{1}^{\prime \prime}-1\right)=b_{1}-1=b$.

If $b \geq 3$ and $d=b$, then some essential arc $\alpha_{i}$ is separating in $F_{i}$, and $F_{n+1}$ must have a disc component. 


\section{Proof of Theorem 1.3.}

Let $X$ be a 3-manifold, and $T$ a 1-manifold properly imbedded in $X$. Let $F$ be a 2-manifold properly imbedded in $X$ transversely to $T$, and $D$ a disc imbedded in $X$ so that $D \cap F$ is a subarc, say $\alpha$, of $\partial D$ so that $D \cap \partial X$ is the complementary arc $\beta=\operatorname{cl}(\partial D-\alpha)$ and so that $D \cap T=\emptyset$. A $\partial$-2-surgery on $F$ along $D$ is such an operation as below. Take a tubular neighbourhood $N(D) \cong D \times[0,1]$ of $D$ so that $N(D) \cap F=\alpha \times[0,1]$, so that $N(D) \cap \partial X=\beta \times[0,1]$ and so that $N(D) \cap T=\emptyset$. Then replace the disc $\alpha \times[0,1]$ on $F$ with the two discs $D \times\{0\} \cup D \times\{1\}$ to obtain a new surface $F^{\prime}$. We call this operation a $\partial-T$-compressing on $F$ along $D$ if $D$ is a $\partial-T$ compressing disc of $F$. We can recover the original surface $F$ from $F^{\prime}$ by a band sum operation as below. Take an arc $\gamma=($ a point $) \times[0,1] \subset D \times[0,1]$, where we take "a point" in the interior of the $\operatorname{arc} \beta$. Then $N(D)$ forms a tubular neighbourhood of $\gamma$ such that $N(D) \cap F^{\prime}=D \times\{0\} \cup D \times\{1\}$ and such that $N(D) \cap T=\emptyset$. Replace the two discs $D \times\{0\} \cup D \times\{1\}$ on $F^{\prime}$ with the disc $\alpha \times[0,1]$ to obtain the original surface $F$.

The next lemma is very clear and we omit the proof.

Lemma 4.1. Let $X$ be a 3-manifold, and $T$ a 1-manifold properly imbedded in $X$. Let $F$ be a 2-manifold properly imbedded in $X$ transversely to $T$. Let $F^{\prime}$ be a 2-manifold obtained by performing a $\partial$-2-surgery and a 2-surgery on $F$ in this order. Then we can obtain $F^{\prime}$ by performing a 2-surgery and a $\partial$-2-surgery on $F$ in this order.

\section{Proof of Theorem 1.3.}

Step 1. First, we isotope $S$ in $(M, T)$ so that $S$ is transverse to the splitting surface $H$. Let $S^{\prime}$ be a 2-manifold transverse to $H$ obtained from $S$ by a mixed sequence of 2 -surgeries, $\partial$-2-surgeries and isotopies in $(M, T)$. The complexity of $S^{\prime}$ is the multi-set of integers $\gamma\left(S^{\prime}\right)=\{|s \cap H| ; s$ is a component of $S^{\prime}$, where "multi-set" may contain the same integers redundantly. We order finite multi-set of integers as follows: Arrange integers in each multiset in monotonically non-increasing order, then compare the ordered multisets lexicographically. We choose $S^{\prime}$ so that $\gamma\left(S^{\prime}\right)$ is minimum over all 2 -manifolds which are obtained by a mixed sequence of 2-surgeries, $\partial-2$ surgeries and isotopies in $(M, T)$ and which have no boundary component forming an inessential loop on $\partial_{-} C_{1}-T$ or $\partial_{-} C_{2}-T$.

Step 2. Let $C_{1}$ and $C_{2}$ be compression bodies obtained by cutting $M$ along $H$. Suppose, for a contradiction, that for $i=1$ or 2 the 2-manifold $S_{i}^{\prime}=S^{\prime} \cap C_{i}$ is $T_{i}$-compressible in $\left(C_{i}, T_{i}\right)$ where $T_{i}=T \cap C_{i}$. Then there is a $T_{i}$-compressing disc $D$ of $S_{i}^{\prime}$. Let $R$ be the component of $S^{\prime}$ such that $\partial D \subset R$. There is a disc $D^{\prime}$ on $R$ such that $\partial D^{\prime}=\partial D$, since $R$ is a disc or sphere. 
Then both $D^{\prime}$ and $\operatorname{cl}\left(R-D^{\prime}\right)$ must intersect $H$ since $D$ is a $T_{i}$-compressing disc. In fact, if $R$ is a $T$-compressing disc of $\partial_{-} C_{i}$ and if $\operatorname{cl}\left(R-D^{\prime}\right)$ is an annulus disjoint from $H$, then $\left(R-D^{\prime}\right) \cup D$ is a disc properly imbedded in $C_{i}-T_{i}$, and $\partial R \cap \partial_{-} C_{i}$ is an inessential simple closed curve in $\partial_{-} C_{i}-T$, which is a contradiction.

If the sphere $D \cup D^{\prime}$ bounds a ball disjoint from $T$ in $M$, then we isotope $D^{\prime}$ onto $D$. Otherwise, we perform a 2-surgery along $D$ on $S^{\prime}$.

In both cases the complexity $\gamma\left(S^{\prime}\right)$ decreases, which is a contradiction.

Step 3. Suppose, for a contradiction, that $S_{1}^{\prime}$ is $T_{1}$ - $\partial$-compressible in $\left(C_{1}, T_{1}\right)$. Then there is a $T_{1}$ - $\partial$-compressing disc $D$ of $S_{1}^{\prime}$.

Suppose that $D$ is incident to $\partial_{-} C_{1}$. Then we perform a $\partial$-2-surgery on $S$ along $D$ to obtain a 2-manifold $S^{\prime \prime}$ with smaller complexity. If $S^{\prime \prime}$ has a boundary component which is inessential on $\partial_{-} C_{1}-T$ and bounds a disc $D^{\prime \prime}$ in $\partial_{-} C_{1}-T$. Then $D \cup D^{\prime \prime}$ forms a $T_{1}$-compressing disc of $S_{1}^{\prime}$, which is a contradiction. Hence $S^{\prime \prime}$ does not have a boundary component which is inessential on $\partial_{-} C_{1}-T$. This is again a contradiction to the minimality of the complexity.

Hence $D$ is incident to $\partial_{+} C_{1}=H$. We isotope $S_{1}^{\prime}$ near the arc $\alpha=S_{1}^{\prime} \cap D$ along the disc $D$. Then a band neighbourhood $N(\alpha)$ of $\alpha$ in $S_{1}^{\prime}$ is pushed into $C_{2}$ and remainder of $S_{1}^{\prime}$ in $C_{1}$ is homeomorphic to the 2-manifold obtained from $S_{1}^{\prime}$ by cutting along $\alpha$. This 2-manifold is $T_{1}$-incompressible in $\left(C_{1}, T_{1}\right)$ since $S_{1}^{\prime}$ is $T_{1}$-incompressible. We repeat this operation until the resultant surface is $T_{1}$ - $\partial$-incompressible. Let $S^{*}$ be the resulting 2-manifold imbedded in $M$. Since $S^{*} \cap \partial_{-} C_{1}=S^{\prime} \cap \partial_{-} C_{1}$ consists of essential loops on $\partial_{-} C_{1}-T$, $S^{*} \cap C_{1}$ consists of spheres, vertical annuli and discs whose boundaries are in $\partial_{+} C_{1}$ by Lemma 2.4 .

Step 4. By applying Lemma 3.1 to every component of $S_{1}^{\prime}$, we can see that $\gamma\left(S^{*}\right)<\gamma\left(S^{\prime}\right)$ if $S_{1}^{\prime}$ contains a $T_{1}$ - $\partial$-compressible component which does not meet $\partial_{-} C_{1}$. This contradicts that $\gamma\left(S^{\prime}\right)$ is minimal since $S^{*}$ is isotopic to $S^{\prime}$ in $(M, T)$.

Hence every $T_{1}-\partial$-compressible component of $S_{1}^{\prime}$ meets $\partial_{-} C_{1}$. Let $Q$ be such a component of $S_{1}^{\prime}$. Then $\gamma\left(S^{*}\right)=\gamma\left(S^{\prime}\right)$ and $S^{*} \cap C_{1}$ contains a component which is a subdisc of $Q$ by Lemma 3.1 (2) and the minimality of $\gamma\left(S^{\prime}\right)$. Hence $S^{*} \cap C_{2}$ contains a component which is not a disc and does not meet $\partial_{-} C_{2}$. The 2-manifold $S^{*} \cap C_{2}$ is $T_{2}$-incompressible by the same argument as in Step 2, and $T_{2}$ - $\partial$-compressible by Lemma 2.4. Then we perform operation as in Step 3 on $S^{*} \cap C_{2}$, and obtain a contradiction to the minimality of $\gamma\left(S^{\prime}\right)=\gamma\left(S^{*}\right)$ by Lemma 3.1 (1).

Step 5 . Hence $S_{1}^{\prime}$ is $T_{1}$ - $\partial$-incompressible in $\left(C_{1}, T_{1}\right)$, and similarly we can show that $S_{2}^{\prime}$ is $T_{2}$ - $\partial$-incompressible in $\left(C_{2}, T_{2}\right)$. Then by Lemma $2.4 S_{i}^{\prime}$ consists of spheres, vertical annuli and discs with their boundary in $\partial_{+} C_{1}$. 
Lemma 4.1 implies that we can obtain $S^{\prime}$ from $S$ by a sequence of isotopies, followed by a sequence of 2-surgeries, followed by a sequence of $\partial$-2-surgeries. Hence by a sequence of band sum operations along arcs on $\partial_{-} C_{1}$ and $\partial_{-} C_{2}$ we can obtain a 2-manifold $\hat{S}$ from $S^{\prime}$ such that $\partial \hat{S}=\partial S$ and such that $\hat{S}$ can be obtained from $S$ by a sequence of isotopies, followed by a sequence of 2-surgeries. Note that these band sum operations are performed along arcs connecting distinct disc components. Let $\hat{S}^{\prime}$ be a 2-manifold obtained from $S^{\prime}$ by a band sum operation along an arc $\gamma$ connecting distinct disc components of $S^{\prime}$. We assume without loss of generality that $\gamma$ is on $\partial_{-} C_{1}$. We can retake the structure $C_{1} \cong Y \cup V$, where $Y$ is homeomorphic to $\partial_{-} C_{1} \times[0,1]$, so that the $\operatorname{disc} Q=\gamma \times[0,1]$ is disjoint from $V$. A standard innermost loop and outermost arc argument allows us to retake $Q$ to be disjoint from the other component of $S_{1}^{\prime}$. We perform a band sum operation on $S^{\prime}$ along $\gamma$ and obtain a disc intersecting $H$ in two loops. We then isotope the band along the disc $Q$, to obtain a disc intersecting $H$ in a single loop. We can retake the structure $C_{1} \cong Y \cup V$ so that the annulus components of $\hat{S}^{\prime} \cap C_{1}$ are vertical. Repeating such operations, we can isotope $\hat{S}$ as in the conclusion of Theorem 1.3. This completes the proof of Theorem 1.3.

Proof of Corollary 1.4. Suppose, for a contradiction, that a component $F$ of $\partial M$ is $T$-compressible in $(M, T)$. Let $C_{1}$ and $C_{2}$ be the compression bodies obtained by cutting $M$ along $H$, and $T_{i}=T \cap C_{i}$ for $i=1$ and 2 . We can assume that witout loss of generality that $\partial_{-} C_{1}$ contains $F$. Let $D$ be a $T$-compressing disc of $F$. Applying Theorem 1.3 we obtain a $T$-compressing disc $D^{\prime}$ of $F$ such that $D^{\prime}$ meets $H$ in a single simple closed curve which is essential on $H-T$. Then $D_{2}=D^{\prime} \cap C_{2}$ is a $T$-compressing disc of $H$. Moreover by Lemma 2.2, we can take a complete disc system $\mathcal{D} \subset C_{1}$ for $\left(\tilde{C}_{1}, T_{1}\right)$ so that $\left(D^{\prime} \cap C_{1}\right) \cap \mathcal{D}=\emptyset$. The complete disc system $\mathcal{D}$ is non-empty since $H$ is not $T$-parallel to $F$ in $\tilde{M}$. Hence we can take a $T$-compressing disc $D_{1}$ of $H$ near $\mathcal{D}$ in $C_{1}$. (In fact, if $\mathcal{D}$ consists of cancelling discs only, then we take a small neighbourhood $N$ of the cancelling disc $Q_{1}$ of some $\partial_{+}$-parallel arc $t$ of $T_{1}$, and the disc $\operatorname{cl}\left(\partial N-\partial_{+} C_{1}\right)$ gives the desired disc $D_{1}$. In this case, the boundary loop $\partial D_{1}$ is essential in $H-T$ since $H-T$ contains an essential loop $\partial D_{2}$ disjoint from $\partial Q_{1}$.) Note that $\partial D_{1} \cap \partial D_{2}=\emptyset$ because $\mathcal{D} \cap D^{\prime}=\emptyset$. Hence $H$ is weakly $T$-reducible, which is a contradiction.

\section{Proof of Theorem 1.1.}

It is very clear that $\partial_{-} C$ is $T$-incompressible for any pair of a compression body $C$ and trivial $\operatorname{arcs} T$.

Lemma 5.1. Let $C$ be a compression body, and $T$ trivial arcs in $C$. The boundary $\partial C$ is $T$-incompressible in $(C, T)$ if and only if it satisfies one of 
the two conditions (1) and (2) below. Otherwise, $\partial_{+} C$ is T-compressible in $(C, T)$.

(1) $\tilde{C}$ is homeomorphic to $\partial_{-} \tilde{C} \times I$ and $T$ consists of some number, perhaps 0 of vertical arcs, or

(2) $\tilde{C}$ is a ball and $T$ consists of 0 or one $\partial_{+}$-parallel arc.

For the definition of $\tilde{C}$, see the sentence right before Lemma 2.2.

Proof. The 'if' part is very clear and we omit the proof. We show the 'only if' part.

Let $Z=\operatorname{cl}(\tilde{C}-C)$ the disjoint union of the balls. There is a homeomorphism $\tilde{C} \cong Y \cup V$ where $Y$ is a ball or homeomorphic to $\partial_{-} \tilde{C} \times I$ and $V$ is a disjoint union of 1-handles. If $V \neq \emptyset$, then we can take a cocore disc $D$ of a 1-handle of $V$. We can isotope $D$ so that $D \cap(T \cup Z)=\emptyset$. Then $D$ is a $T$-compressing disc of $\partial_{+} C$ in $(C, T)$.

If $V=\emptyset$, then $\tilde{C}$ is a ball or homeomorphic to $\partial_{-} \tilde{C} \times I$. Suppose that $T$ contains a $\partial_{+}$-parallel arc $t$. Let $Q$ be a cancelling disc of $t$. We take a small neighbourhood $N(Q)$. Then the disc $Q^{\prime}=\operatorname{cl}\left(\partial N(Q)-\partial_{+} C\right)$ cuts off the ball $N(Q)$ containing $t$ from $C$. If $\partial Q^{\prime}$ is inessential on $\partial_{+} C-T$, then $\partial_{+} C$ is a sphere and $T=\{t\}$, and hence $\tilde{C}$ is a ball.

Lemma 5.2. Let $M$ be a compact connected orientable 3-manifold, and $T$ 1-manifold properly imbedded in $M$. Suppose that a strongly $T$-irreducible Heegaard splitting $(M, T)=\left(C_{1}, T_{1}\right) \cup_{H}\left(C_{2}, T_{2}\right)$ is given, and that $\partial_{+} C_{1}$ is $T_{1}$-compressible in $\left(C_{1}, T_{1}\right)$. If a component $F$ of $\partial_{-} C_{1}$ has a thinning disc $D$ in $(M, T)$, then $(M, T)$ is homeomorphic to a pair $(V, S)$ of type (2) of Lemma 5.1 with $\partial_{+} V=\partial_{-} C_{1}$.

Proof. Let $\tilde{M}$ be the 3 -manifold obtained by capping off all the spherical boundary components disjoint from $T$ with balls. Let $N(D)$ be a small regular neighbourhood of $D$ in $M$. Then $D^{\prime}=\operatorname{cl}\left(\partial N(D)-\partial_{-} C_{1}\right)$ is a disc which cuts off the ball $N(D)$ containing the arc $D \cap T$ from $(M, T)$. By Corollary $1.4 \partial_{-} C_{1}$ is $T$-incompressible in $(M, T)$. Hence $\partial D^{\prime}$ bounds a disc $D^{\prime \prime}$ disjoint from $T$ in $F$. If the sphere $S=D^{\prime} \cup D^{\prime \prime}$ is not a splitting sphere of $(\tilde{M}, T)$, then it bounds a ball disjoint from $T$ in $\tilde{M}$, and we obtain the desired conclusion. If $S$ is a splitting sphere of $(\tilde{M}, T)$, then by Theorem 1.3 we obtain a disjoint union $S^{\prime}$ of 2 -spheres by 2 -surgeries and isotopy in $(\tilde{M}, T)$ such that $S^{\prime} \cap H=\emptyset$ since $H$ is strongly $T$-irreducible also as a Heegaard splitting of $(\tilde{M}, T)$. These spheres of $S_{\tilde{C}}^{\prime}$ bound balls which are contained in the irreducible compression bodies $\tilde{C}_{1}$ or $\tilde{C}_{2}$ and are disjoint from $T$. Since $S^{\prime}$ is obtained from $S$ by 2 -surgeries and isotopy, $S$ also bounds a ball disjoint from $T$ in $(\tilde{M}, t)$. Hence we obtain the desired conclusion.

Lemma 5.3. Let $M$ be a compact connected orientable 3-manifold, and $T$ a 1-manifold properly imbedded in $M$. Suppose that there is given a multiple 
Heegaard splitting $(M, T)=\left(C_{1}, T_{1}\right) \cup_{\mathcal{H}}\left(C_{2}, T_{2}\right)$ such that $C_{1}$ and $C_{2}$ are compression bodies, $\mathcal{H}_{+}=H_{+}=\partial_{+} C_{1}=\partial_{+} C_{2}$, and $\mathcal{H}_{-}$contains a component $H_{-}$of $\partial_{-} \tilde{C}_{1} \cap \partial_{-} \tilde{C}_{2}$. If $\partial_{+} C_{1}$ is $T_{1}$-incompressible in $\left(C_{1}, T_{1}\right)$, then for $i=1$ and $2, C_{i} \cong \partial_{-} C_{i} \times I-($ balls $)$ and $T_{i}$ consists of vertical arcs.

Proof. Since $\left(C_{1}, T_{1}\right)$ is of type (1) of Lemma 5.1, $C_{1} \cong \partial_{-} \tilde{C}_{1} \times I-($ balls $)$ and $T_{1}$ consists of vertical arcs, where $\partial_{-} \tilde{C}_{1}=H_{-}$and $\partial_{+} C_{1}=\partial_{-} \tilde{C}_{1} \times\{1\}$. Note that $\operatorname{genus}\left(\partial_{+} C_{2}\right)=\operatorname{genus}\left(\partial_{+} C_{1}\right)=\operatorname{genus}\left(\partial_{-} \tilde{C}_{1}\right)$. Hence $\operatorname{genus}\left(H_{-}\right)=$ genus $\left(\partial_{+} C_{2}\right)$, the components of $\partial_{-} C_{2}$ other than $H_{-}$are spheres, and $C_{2} \cong$ $H_{-} \times I-$ (balls). Since $\left|T \cap \partial_{+} C_{1}\right|=\left|T \cap \partial_{-} \tilde{C}_{1}\right|$, it follows that $\left|T \cap \partial_{+} C_{2}\right|=$ $\left|T \cap H_{-}\right|$and that $T_{2}$ consists of vertical arcs connecting $\partial_{+} C_{2}$ and $H_{-}$. Thus $\partial_{-} \tilde{C}_{2}=H$.

Let $M$ be a compact connected orientable 3 -manifold, and $T$ a 1-manifold properly imbedded in $M$. Let $\mathcal{H}$ be a multiple Heegaard splitting of $(M, T)$. The surfaces of $\mathcal{H}$ divide $M$ into compression bodies $C_{1}, \ldots, C_{n}$. The arcs $T_{i}=T \cap C_{i}$ are trivial in $C_{i}$. Let us remember that $W_{i j}=C_{i} \cup C_{j}$ is a component of the 3 -manifold obtained by cutting $M$ along $\mathcal{H}_{-}$, where $\partial_{+} C_{i}=\partial_{+} C_{j}=H_{i j} \subset \mathcal{H}_{+}$. Let $T_{i j}=T \cap W_{i j}$.

Proposition 5.4. Suppose that for each $1 \leq i \leq n$ either:

(1) $\partial_{+} C_{i}$ is $T_{i}$-compressible in $\left(C_{i}, T_{i}\right)$ or

(2) $\left(C_{i}, T_{i}\right)$ is of type (1) of Lemma 5.1 and

(a) the surface $\partial_{-} \tilde{C}_{i}$ is a component of $\partial M$ or

(b) for some $C_{j}, \partial_{+} C_{i}=\partial_{+} C_{j}$ and $\partial_{-} \tilde{C}_{i} \cap \partial_{-} \tilde{C}_{j} \neq \emptyset$ or

(3) $\left(C_{i}, T_{i}\right)$ is of type (2) of Lemma 5.1.

Moreover, suppose that the splitting $H_{i j}$ of $\left(W_{i j}, T_{i j}\right)$ is strongly T-irreducible for all components of $\mathcal{H}_{+}$. Then $\mathcal{H}_{-}$is $T$-incompressible in $(M, T)$. In addition, a component $F$ of $\partial M$ is $T$-incompressible if the pair $\left(C_{k}, T_{k}\right)$ containing $F$ is not of type (1) of Lemma 5.1.

Proof. Suppose, for a contradiction, that a component $H$ of $\mathcal{H}_{-}$is $T$-compressible in $(M, T)$. Let $D$ be a $T$-compressing disc of $H, C_{i}$ the compression body containing a collar of $\partial D$ in $D$, and $C_{j}$ the compression body such that $\partial_{+} C_{i}=\partial_{+} C_{j}$. Then by applying an innermost disc argument on the curves of $\mathcal{H}_{-} \cap D$ and replacing $H$ and $C_{i}$ if necessary, we can assume that $D \subset W_{i j}$. Since the spheres $\partial_{-} C_{i}-\partial_{-} \tilde{C}_{i}$ are $T$-incompressible, $H \subset \partial_{-} \tilde{C}_{i}$. The boundary $\partial_{+} C_{i}$ is $T_{i}$-compressible in $\left(C_{i}, T_{i}\right)$ or the pair $\left(C_{i}, T_{i}\right)$ is of type $(2 \mathrm{~b})$ since $\emptyset \neq \partial_{-} \tilde{C}_{i}=H \subset \mathcal{H}_{-}$. In the former case, by Corollary 1.4 the splitting $H_{i j}$ of $\left(W_{i j}, T_{i j}\right)$ is weakly $T$-reducible, which is a contradiction. In the latter case, by Lemma $5.3, H$ is clearly $T$-incompressible in $(M, T)$.

Let $F$ be a component of $\partial M$, and $C_{k}$ the compression body containing $F$. Suppose, for a contradiciton, that $F$ is $T$-compressible in $(M, T)$ and $\partial_{+} C_{k}$ is $T_{k}$-compressible in $\left(C_{k}, T_{k}\right)$. Let $D$ be a $T$-compressing disc of $F$, 
$C_{l}$ the compression body such that $\partial_{+} C_{l}=\partial_{+} C_{k}$. We can assume that $D \subset W_{k l}$ since $\mathcal{H}_{-}$is $T$-incompressible. This contradicts Corollary 1.4.

Lemma 5.5. Suppose that the splitting $\mathcal{H}$ is slim. Then for each $1 \leq i \leq n$, $\left(C_{i}, T_{i}\right)$ is of type either (1), (2) or (3) of Proposition 5.4.

Proof. Suppose, for a contradiction, that there is a pair $\left(C_{i}, T_{i}\right)$ such that $\partial_{+} C_{i}$ is $T_{i}$-incompressible in $\left(C_{i}, T_{i}\right)$ and $\left(C_{i}, T_{i}\right)$ is not of type (2) or (3) of Proposition 5.4. Then $C_{i} \cong \partial_{-} C_{i} \times I-$ (balls) and $T_{i}$ is empty or consists of vertical arcs by Lemma 5.1. Let $C_{j}$ be the compression body such that $\partial_{+} C_{j}=\partial_{+} C_{i}$. Note that $\partial_{-} \tilde{C}_{i} \cap \partial_{-} \tilde{C}_{j}=\emptyset$ and $\partial_{-} \tilde{C}_{i} \cap \partial M=\emptyset$ since $\left(C_{i}, T_{i}\right)$ is not of type (2). There is a compression body $C_{k}$ such that $\partial_{-} \tilde{C}_{i} \subset \partial_{-} C_{k}$ $(k \neq j)$. Then $C_{*}=C_{j} \cup_{F} C_{i} \cup_{G} C_{k}$, where $F=\partial_{+} C_{i}=\partial_{+} C_{j}$ and $G=\partial_{-} \tilde{C}_{i} \subset \partial_{-} C_{k}$, is a compression body with $\partial_{+} C_{*}=\partial_{+} C_{k}$ and $\partial_{-} C_{*}=$ $\left(\left(\partial_{-} C_{k} \cup \partial_{-} C_{i}\right)-\partial_{-} \tilde{C}_{i}\right) \cup \partial_{-} C_{j}$, and $T_{*}=T_{j} \cup T_{i} \cup T_{k}$ is trivial in $C_{*}$. Hence $\mathcal{H}-\left(\partial_{-} \tilde{C}_{i} \cup \partial_{+} C_{i}\right)$ is a multiple Heegaard splitting of $(M, T)$. This contradicts that $\mathcal{H}$ is slim.

Lemma 5.6. Suppose that the splitting $\mathcal{H}$ is slim. Then no component $H$ of $\mathcal{H}_{-}$cuts off a pair $(V, S)$ such that $V$ is a compression body with $H=\partial_{+} V$, and $\partial_{-} V \subset \mathcal{H}$ such that $S$ is a disjoint uinon of trivial arcs in $V$ and such that some numbers, perhaps 0 of pairs of components of $\partial_{-} V$ are amalgamated and the other components of $\partial_{-} V$ is contained in $\partial M$.

Proof. Suppose that there is such a component $H \subset \mathcal{H}_{-}$. Let $C_{i}$ be the compression body such that $V \cap C_{i}=\partial_{+} V \cap \partial_{-} C_{i}=H$. Then $\left(C_{i}, T_{i}\right) \cup(V, S)$ is a pair of compression body and trivial arcs in it after cut open along the amalgamated components of $\partial_{-} V$. Hence $\mathcal{H}-\left((\mathcal{H} \cap V)-\partial_{-} V\right)$ is a multiple Heegaard splitting of $(M, T)$. This contradicts that $\mathcal{H}$ is slim.

Lemma 5.7. Suppose that the splitting $\mathcal{H}$ is slim. Then $\mathcal{H}_{-}$has no thinning disc in $(M, T)$.

Proof. Suppose, for a contradiction, that some component $H$ of $\mathcal{H}_{-}$has a thinning disc $Q$. We can isotope $Q$ slightly so that $Q \cap \mathcal{H}_{-}$consists of loops, the $\operatorname{arc} \beta=\operatorname{cl}(\partial Q-T)$ and properly imbedded arcs with endpoints in $Q \cap T$.

Since $\mathcal{H}_{-}$is $T$-incompressible by Proposition 5.4 and Lemma 5.5, a standard innermost loop argument allows us to retake $Q$ so that $Q$ intersects $\mathcal{H}_{-}$in arcs only. Moreover, a standard outermost arc argument allows us to retake $H$ and $Q$ so that $Q \cap \mathcal{H}_{-}=Q \cap H=\operatorname{cl}(\partial Q-T)=\beta$.

Then we can take a collar $\partial Q \times I$ of $\partial Q$ in $Q$ so that $\beta \times I$ is contained in some compression body $C_{i}$. Let $C_{j}$ be another compression body such that $\partial_{+} C_{j}=\partial_{+} C_{i}$.

We first suppose that $\partial C_{i}$ is $T_{i}$-compressible in $\left(C_{i}, T_{i}\right)$. Then by Lemma $5.2,\left(W_{i j}, T_{i j}\right)$ is homeomorphic to a pair $(V, S)$ of type (2) of Lemma 5.1 with $\partial_{+} V=H$. Let $C_{k},(k \neq i$ nor $j)$ be the compression body such 
that $H \subset \partial_{-} C_{k}$. (Note that $k \neq i$ and $k \neq j$ follows from the conditions $|H \cap T|=2$ and $\partial_{-} V \cap T=\emptyset$.) Then $C_{k} \cup W_{i j}$ is a compression body and $T_{k} \cup T_{i j}$ is a trivial set of arcs in it. Hence $\mathcal{H}-\left(H \cup H_{i j}\right)$ is a multiple Heegaard splitting of $(M, T)$, which contradicts that $\mathcal{H}$ is slim.

Suppose secondly that $\partial C_{i}$ is $T_{i}$-incompressible in $\left(C_{i}, T_{i}\right)$. Then by Lemma 5.5 this pair $\left(C_{i}, T_{i}\right)$ is of type (2b) of Proposition 5.4 since $\left(\partial_{-} C_{i} \cap\right.$ $\left.\mathcal{H}_{-}\right) \supset H$ and $H \cap T \neq \emptyset$. Then by Lemma $5.3, H$ never has a thinning disc. This is a contradiction.

Proposition 5.4 and Lemmas 5.5, 5.6 and 5.7 together complete the proof of Theorem 1.1.

\section{Proof of Theorem 1.2.}

In general, let $F$ be a closed (possibly disconnected) 2-manifold. Let $\alpha$ be disjoint union of loops on $F$. Then let $\sigma(F, \alpha)$ denote the closed 2-manifold obtained by cutting $F$ along $\alpha$ and capping off the resulting boundary circles with discs.

Let $F$ be a closed (possibly disconnected) 2-manifold with punctured points. Let $w(F)$ be the multi-set of pairs as the definition of width in Section 1 regarding the punctured points as intersection points with $T$. We define $\mu(F)$ as multi-set of pairs obtained from $w(F)$ by deleting all the $(0,0)$ elements. We order $\mu(F)$ in the same way as width.

Proof of Theorem 1.2. The proof of Theorem 1.2 is very similar to that of Theorem 3.1 in $[\mathbf{C}-\mathbf{G}]$. First we describe how to take the disc systems $\mathcal{D}, \mathcal{D}^{\prime}, \mathcal{E}, \mathcal{E}^{\prime}$ in the proof in $[\mathbf{C}-\mathbf{G}]$.

Let $W$ and $W^{\prime}$ be the compression bodies obtained by cutting $M$ along $H$. Then $T \cap W$ and $T \cap W^{\prime}$ are trivial arcs in the compression bodies $W$ and $W^{\prime}$.

Claim 6.1. Let $D$ and $D^{\prime}$ be $T$-compressing discs of $H$ in $W$ and $W^{\prime}$ respectively such that $\partial D \cap \partial D^{\prime}=\emptyset$. Then $\mu\left(\sigma\left(H, \partial D \cup \partial D^{\prime}\right)\right)<\mu(\sigma(H, \partial D))$, $\mu\left(\sigma\left(H, \partial D^{\prime}\right)\right)$.

Proof of Claim 6.1. Suppose, for a contradiction, that $\mu\left(\sigma\left(H, \partial D \cup \partial D^{\prime}\right)\right)$ $=\mu(\sigma(H, \partial D))$. Then there is a sphere component $Q$ of $\sigma\left(H, \partial D \cup \partial D^{\prime}\right)$ such that $Q \cap T=\emptyset$ and $Q$ contains a copy of $D^{\prime}$. If $Q$ does not contain a copy of $D$ and contains only single copy of $D^{\prime}$, then we have a contradiction to the fact that $D^{\prime}$ is a $T$-compressing disc of $H$. If $Q$ does not contain a copy of $D$ and contains two copies of $D^{\prime}$, then $H$ is a torus which does not contain $\partial D$. This is also a contradiction. Hence $Q$ contains a copy of $D$. There is a loop in $Q$ which separates copies of $D$ and those of $D^{\prime}$. Then the loop bounds discs disjoint from $T$ near $Q$ in both $W$ and $W^{\prime}$. These discs are $T$-compressing disc of $H$ because $D$ and $D^{\prime}$ are $T$-compressing disc of $H$. This contradicts to the condition that $H$ is $T$-irreducible. 
Since $H$ is weakly $T$-reducible, by Claim 6.1 there are non-empty disjoint unions of discs $\mathcal{D}, \mathcal{D}^{\prime}$ properly imbedded in $W, W^{\prime}$ respectively such that:

(1) The discs of $\mathcal{D}$ and $\mathcal{D}^{\prime}$ are $T$-compressing discs of $H$,

(2) $\partial \mathcal{D} \cap \partial \mathcal{D}^{\prime}=\emptyset$,

(3) $\mu\left(\sigma\left(H, \partial \mathcal{D} \cup \partial \mathcal{D}^{\prime}\right)\right)<\mu(\sigma(H, \partial \mathcal{D})), \mu\left(\sigma\left(H, \partial \mathcal{D}^{\prime}\right)\right)$

and $\mu\left(\sigma\left(H, \partial \mathcal{D} \cup \partial \mathcal{D}^{\prime}\right)\right)$ is minimal subject to these conditions.

We untelescope $\{H\}$ along these discs as in the proof of Lemma 2.3. That is, let $C_{1}^{\prime}=\operatorname{cl}(W-N(\mathcal{D}))$ and $C_{4}^{\prime}=\operatorname{cl}\left(W^{\prime}-N\left(\mathcal{D}^{\prime}\right)\right)$. We take a small collar $N\left(\partial_{+} C_{k}^{\prime}\right)$ in $C_{k}^{\prime}$ for $k=1$ and 4 . Let $C_{k}=\operatorname{cl}\left(C_{k}^{\prime}-N\left(\partial_{+} C_{k}^{\prime}\right)\right)$ for $k=1$ and 4. Let $C_{2}=N\left(\partial_{+} C_{1}\right) \cup N\left(\mathcal{D}^{\prime}\right)$ and $C_{3}=N\left(\partial_{+} C_{4}\right) \cup N(\mathcal{D})$. These are disjoint unions of compression bodies such that $\partial_{+} C_{1}=\partial_{+} C_{2}$, $\partial_{+} C_{3}=\partial_{+} C_{4}$ and $\partial_{-} C_{2}=\partial_{-} C_{3} \cong \sigma\left(H, \partial \mathcal{D} \cup \partial \mathcal{D}^{\prime}\right)$. Let $H_{0}$ denote this 2-manifold $\partial_{-} C_{2}=\partial_{-} C_{3}$.

Since $H$ is connected, there is a component $F$ of $H_{0}$ such that $F \cap$ (int $W) \neq \emptyset$ and $F \cap\left(\right.$ int $\left.W^{\prime}\right) \neq \emptyset$. Then we can show that $F$ is not a 2-sphere disjoint from $T$ by taking a loop separating the copies of discs of $\mathcal{D}$ and those of $\mathcal{D}^{\prime}$ as in the proof of Claim 6.1.

Suppose, for a contradiction, that $F$ is $T$-compressible, say in $\left(C_{1}, T_{1}\right) \cup$ $\left(C_{2}, T_{2}\right)$, where $T_{i}=T \cap C_{i}$. Let $\Gamma$ be the union of the cocore arcs of the 2-handles $N(\mathcal{D})$. We extend $\Gamma$ by jointing vertical arcs in the collar neighbourhood $N\left(\partial_{+} C_{1}^{\prime}\right) \cong \partial_{+} C_{1}^{\prime} \times I$ so that $\Gamma$ has all the endpoints in $\partial_{+} C_{1}$. Then $C_{1} \cup N(\Gamma)$ is ambient isotopic to $W$ in $(M, T)$.

The surface $F$ has a $T$-compressing disc $D$. We can assume without loss of generality that $D$ is contained in $C_{1} \cup C_{2}$ rather than $C_{3} \cup C_{4}$. Theorem 1.3 implies that there is a $T$-compressing disc $D$ of $F$ such that $D_{0}=D \cap C_{1}$ is a $T$-compressing disc of $\partial_{+} C_{1}$ in $\left(C_{1}, T_{1}\right)$. Let $S=\Gamma \cap C_{2}$. Note that $T_{2} \cup S$ is a union of vertical arcs in $C_{2}$. Possibly $D$ is not vertical with respect to the product structure $N\left(\partial_{+} C_{1}^{\prime}\right) \cong \partial_{+} C_{1}^{\prime} \times I$. But we can retake $D$ to be disjoint from the arcs $S$ as below. We take a disjoint union of annuli $A$ propery imbedded in $C_{2}$, one for every component of $\partial_{-} C_{2}$, so that $\left(T_{2} \cup S\right) \subset A$, and that it is vertical in $\partial_{+} C_{1}^{\prime} \times I$. Moreover, for every non-sphere component $H_{0}^{\prime}$ of $\partial_{-} C_{2}$, we can take $A$ so that the boundary loop $A \cap H_{0}^{\prime}$ is essential on $H_{0}^{\prime}$. We can retake $D$ so that it intersects $A$ transversely and that $D \cap A$ contains no inessential loop on $A$. Since $D$ does not intersect $T$, we can isotope $S$ on $A$ so that it does not intersect arc components of $D \cap A$. Let $\ell$ be an essential loop of $D \cap A$ on $A$ such that $\ell$ is the nearest to $\partial_{-} C_{2}$. Let $H_{0}^{\prime}$ be the component of $\partial_{-} C_{2}$ which is incident to the annulus containing the loop $\ell$. Note that $H_{0}^{\prime}$ is disjoint from $T$. The loop $\ell$ divides $D$ into a disc $D_{D}$ and an annulus $A_{D}$, and does a component of $A$ into two annuli, one of which, say $A_{A}$, is incident to $\partial_{-} C_{2}$. If $H_{0}^{\prime}$ is not a sphere, then we substitute $A_{A}$ with $A_{D}$ on $D$. An adequate small isotopy of the disc $D_{D} \cup A_{A}$ decreases the number of intersection points of $D \cap S$. 
If $H_{0}^{\prime}$ is a sphere, then a boundary loop of $A_{A}$ divides $H_{0}^{\prime}$ into two discs $Q_{1}$ and $Q_{2}$. One of the discs $A_{D} \cup A_{A} \cup Q_{1}$ and $A_{D} \cup A_{A} \cup Q_{2}$ intersects $S$ in smaller number of points than $D$ after an adequate small isotopy. Repeating such operations, we can retake $D$ to be disjoint from the $\operatorname{arcs} S$.

Let $\tilde{C}_{2}$ be the compression body obtained from $C_{2}$ by capping off all the spheres of $\partial_{-} C_{2}$ disjoint from $T_{2} \cup S$ with balls. By Lemma 2.2, there is a complete disc system $\mathcal{E}^{\prime}$ of $\left(\tilde{C}_{2}, T_{2} \cup S\right)$ such that $\mathcal{E}^{\prime} \cap D=\emptyset$. Let $\mathcal{E}=(\mathcal{D} \cap N(\Gamma)) \cup D_{0}$. The unions of discs $\mathcal{E}$ and $\mathcal{E}^{\prime}$ can be regarded as unions of $T$-compressing discs of $H$ imbedded in $W$ and $W^{\prime}$ respectively since $C_{1} \cup N(\Gamma)$ is ambient isotopic to $W$ in $(M, T)$. Then we can see that these systems of discs $\mathcal{E}$ and $\mathcal{E}^{\prime}$ violate the minimality of $\mu\left(H_{0}\right)$ as below. The surface $\sigma\left(H, \partial \mathcal{E} \cup \partial \mathcal{E}^{\prime}\right)$ is homeomorphic to $\sigma\left(H_{0}, \partial D\right)$ modulo 2-spheres disjoint from $T$ because $\mathcal{E}=(\mathcal{D} \cap N(\Gamma)) \cup D_{0}$ and because $\mathcal{E}^{\prime}$ is a complete disc system of $\left(\tilde{C}_{2}, T_{2}\right)$. Since the disc $D$ is a $T$-compressing disc of $H_{0}$, we have $\mu\left(\sigma\left(H, \partial \mathcal{E} \cup \partial \mathcal{E}^{\prime}\right)\right)<\mu\left(H_{0}\right)$. If $\mu\left(\sigma\left(H, \partial \mathcal{E} \cup \partial \mathcal{E}^{\prime}\right)\right)=\mu(\sigma(H, \partial \mathcal{E}))$ or $\mu\left(\sigma\left(H, \partial \mathcal{E} \cup \partial \mathcal{E}^{\prime}\right)\right)=\mu\left(\sigma\left(H, \partial \mathcal{E}^{\prime}\right)\right)$, then we have a contradiction as in the proof of Claim 6.1. Thus we obtain a contradiction to the minimality of $\mu\left(H_{0}\right)$.

Let $\Lambda$ be the union of the cocore arcs of the 2-handles $N(\mathcal{D}), N\left(\mathcal{D}^{\prime}\right)$. We can recover the Heegaard splitting surface $H$ by performing surgeries along $\Lambda$ on $H_{0}$.

In the rest of this proof, we assume that the Heegaard splitting $H$ of $(M, T)$ is not cancellable. Suppose, for a contradiction, that $F$ is a 2 -sphere bounding in $\tilde{M}$ a ball $B$ intersecting $T$ in a trivial arc $t$. Let $\mathcal{H}_{1}$ be the surfaces of $\mathcal{H}_{-} \cap B$, and $H^{\prime}$ the surface obtained by performing surgery on $\mathcal{H}_{1}$ along the arcs $\Lambda \cap B$. Then $H^{\prime}$ gives a Heegaard splitting of $(B, t)$ when it is isotoped slightly into $\operatorname{int} B$. This splitting $H^{\prime}$ is not trivial, i.e., not $T$-parallel to $\partial B$, since $\Lambda \cap B \neq \emptyset$ from the way of taking $F$. Hence by Lemma 2.1 in [H-S2] as below, which derives from Lemma 2.1 in [H-S1], $H^{\prime}$ is cancellable or stabilized. In the former case $H$ is also cancellable, and in the latter case the sphere $\partial N\left(D_{1} \cup D_{2}\right)$ shows that $H$ is $T$-reducible where $D_{1}$ and $D_{2}$ are discs showing that $H^{\prime}$ is stabilized. In both cases we obtain contradictions.

Lemma 2.1 in [H-S2]. Let $B$ be a ball, $t$ a single trivial arc in $B$ and $H^{\prime}$ a Heegaard splitting of $(B, t)$. Then $H^{\prime}$ is either trivial, cancellable or stabilized.

Suppose, for a contradiction, that $F$ is a torus bounding in $\tilde{M}$ a solid torus $V$ intersecting $T$ in a core loop $t$ of $V$. Let $\mathcal{H}_{2}$ be the surfaces of $\mathcal{H}_{-} \cap V$, and $H^{\prime \prime}$ the surface obtained by performing surgery on $\mathcal{H}_{2}$ along the arcs $\Lambda \cap V$. Then $H^{\prime \prime}$ gives a Heegaard splitting of $(V, t)$ when it is isotoped slightly into int $V$. Hence $H^{\prime \prime}$ is cancellable or stabilized by [H-S3, 
Theorem 1.1] below. In the former case $H$ is also cancellable, and in the latter case $H$ is $T$-reducible. In both cases we obtain contradictions.

Theorem 1.1 in [H-S3]. Let $V$ be a solid torus, $t$ a core loop of $V$ and $H^{\prime \prime}$ a Heegaard splitting of $(V, t)$. Then $H^{\prime \prime}$ is either cancellable or stabilized. Moerover, when $\left|H^{\prime \prime} \cap t\right|=2$ and genus $\left(H^{\prime \prime}\right) \geq 2, H^{\prime \prime}$ is stabilized.

In the rest of this proof, we assume that the Heegaard splitting $H$ of $(M, T)$ is not netted. Suppose for a contradiction that $F$ is $T$-parallel to a component of $\partial \tilde{M}$ in $\tilde{M}$. Let $\left(P \cong F \times I, T^{\prime}\right)$ be the parallelism between $F$ and a component of $\partial \tilde{M}$, where $T^{\prime}=T \cap P$ are vertical arcs. Let $\mathcal{H}_{3}$ be the surfaces of $\mathcal{H}_{-} \cap P$, and $H^{\prime \prime \prime}$ the surface obtained by performing surgery on $\mathcal{H}_{3}$ along the arcs $\Lambda \cap P$. Then $H^{\prime \prime \prime}$ gives a Heegaard splitting of $\left(P, T^{\prime}\right)$ when it is isotoped slightly into int $P$. Hence by [H-S2, Proposition 2.3] below, $H^{\prime \prime \prime}$ is trivial, cancellable or stabilized.

Proposition 2.3 in [H-S2]. Let $F$ be a closed connected orientable surface, and $T^{\prime}$ vertical arcs in $F \times I$. Suppose a surface $H^{\prime \prime \prime}$ gives a Heegaard splitting of $(F \times I, T)$. Then $H^{\prime \prime \prime}$ is either trivial, cancellable or stabilized. Here, a trivial Heegaard splitting surface is either (type I) T-parallel to $F \times\{0\}$ or (type II) obtained by performing a tubing operation on $F \times\{0\}$ and $F \times\{1\}$ along a vertical arc disjoint from $T$ and pushing the resulting surface into $\operatorname{int}(F \times I)$.

Since $\Lambda \cap P \neq \emptyset$ from the way of taking $F, H^{\prime \prime \prime}$ is not $T$-parallel to $F$, that is, not trivial of type I. When $H^{\prime \prime \prime}$ is trival of type II, $\mathcal{H}_{3}$ consists of $F$ and a surface $T$-parallel to $F$ in $P$ and $\Lambda \cap P$ consists of a vertical arc. This implies that $H$ is netted, which is a contradiction. When $H^{\prime \prime \prime}$ is cancellable, $H$ is also cancellble, which is a contradiction. At last, when $H^{\prime \prime \prime}$ is stabilized, $H$ is also stabilized, and hence is $T$-reducible by the weakly $T$-reducibility. This is again a contradiction.

\section{References}

[B-O] F. Bonahon and J.-P. Otal, Scindements de Heegaard des espaces lenticulaires, Ann. Sci. Ec. Norm. Sup., 16(4) (1983), 451-466.

[C-G] A.J. Casson and C.McA. Gordon, Reducing Heegaard splittings, Topology Appl., 27 (1987), 275-283.

[D] H. Doll, A generalized bridge number for links in 3-manifolds, Math. Ann., 294 (1992), 701-717.

[F] C. Feist, Results on thin position, Ph.D. thesis, University of California, 1998.

[G] D. Gabai, Foliations and the topology of 3-manifolds, III, J. Differential Geom., 26 (1987), 479-536.

[G-L] C.McA. Gordon and J. Luecke, Knots are determined by their complements, J. Amer. Math. Soc., 2 (1989), 371-415. 
[G-M] L. Grasselli and M. Mulazzani, Genus one 1-bridge knots and Dunwoody manifolds, preprint.

[Hk] W. Haken, Some results on surfaces in 3-manifolds, Studies in Modern Topology (Math. Assoc. Amer., distributed by: Prentice-Hall), Studies in Math., 5 (1968), 34-98.

[Hy1] C. Hayashi, Genus one 1-bridge positions for the trivial knot and cabled knots, Math. Proc. Camb. Phil. Soc., 125 (1999), 53-65.

[Hy2] _ Satellite knots in 1-genus 1-bridge positions, Osaka J. Math., 36 (1999), 203-221.

[Hy3] _ Stable equivalence of Heegaard splittings of 1-submanifolds in 3manifolds, Kobe J. Math., 15 (1998), 147-156.

[Hy4] , 1-genus 1-bridge splittings for knots in the 3-sphere and lens spaces, preprint.

[H-S1] C. Hayashi and K. Shimokawa, Heegaard splittings of the trivial knot, Knots Theory Ramification, 7 (1998), 1073-1085.

[H-S2] Heegaard splittings of trivial arcs in compression bodies, to appear in Knots Theory Ramification.

[H-S3] _ Heegaard splittings of the pair of the solid torus and the core loop, to appear in Rev. Mat. Complut..

[H-K] D.J. Heath and T. Kobayashi, Essential tangle decomposition from thin position of a link, Pacific J. Math., 179 (1997), 101-117.

[Ho] P. Hoidn, On 1-bridge genus of small knots, preprint.

[Ja] W. Jaco, Lectures on Three-Manifold Topology, (CBMS Reg. Conf. Ser., Vol. 43), Providence, RI, Am. Math. Soc., 1980.

[Jo] K. Johannson, On surfaces and Heegaard surfaces, Trans. Amer. Math. Soc., 325(2) (1991), 573-591.

[K] T. Kobayashi, Structures of the Haken manifolds with Heegaard splitting of genus two, Osaka J. Math., 21 (1984), 437-455.

[K-S] T. Kobayashi and O. Saeki, Rubinstein-Scharlemann graphic of 3-manifold as the discriminant set of a stable map, Pacific J. Math., 195(1) (2000), 101-156.

[L-M] M. Lustig and Y. Moriah, Close incompressible surfaces in complement of wide knots and links, Topology Appl., 92 (1999), 1-13.

[M] K. Morimoto, On minimum genus Heegaard splittings of some orientable closed 3-manifolds, Tokyo J. Math., 12(2) (1989), 321-355.

[M-Sa] K. Morimoto and M. Sakuma, On unknotting tunnels for knots, Math. Ann., 289 (1991), 143-167.

[M-S-Y] K. Morimoto, M. Sakuma and Y.Yokota, Examples of tunnel number one knots which have the property ' $1+1=3$ ', Math. Proc. Camb. Phil. Soc., 119 (1996), 113-118.

[M-Sl] K. Morimoto and J. Schultens, Tunnel numbers of small knots do not go down under connected sum, Proc. Amer. Math. Soc., 128 (2000), 269-278.

[O] M. Ochiai, On Haken's theorem and its extension, Osaka J. Math., 20 (1983), 461-468.

[S-S] M. Scharlemann and J. Schultenz, The tunnel number of the connect sum of $n$ knots is at least $n$, Topology, 38 (1999), 265-270. 
[S-T] M. Scharlemann and A. Thompson, Thin position for 3-manifold, Contemp. Math., 164 (1994), 231-238.

[Sb] H. Schubert, Ueber eine numerische Knoteninvariante, Math. Z., 61 (1954), 245288.

[Sl] J. Schultens, Additivity of tunnel number for small knots, preprint

[S-Ki] H.J. Song and S.H. Kim, Dunwoody 3-manifolds and (1,1)-decomposible knots, preprint.

[S-Ko] H.J. Song and K.H. Ko, Spatial $\theta$-curve associated with Dunwoody $(1,1)$ decomposable knots, preprint.

[T] A. Thompson, Thin position and bridge number for knots in the 3-sphere, Topology, 36 (1996), 505-507.

Received February 15, 1999. The first author was partially supported by Grant-in-Aid for Scientific Research (No. 10740039), Ministry of Education, Science and Culture. The second author was partially supported by Fellowship of the Japan Society for the Promotion of Science for Japanese Junior Scientists.

GaKushuin University

1-5-1 Mejiro Toshima-KU

TOKYO, 171-8588

JAPAN

E-mail address: Chuichiro.Hayashi@gakushuin.ac.jp

TOHOKU UNIVERSITY

2-1-1 Katahira AOBA-KU

SENDAI 980-8577

JAPAN

E-mail address: koya@math.is.tohoku.ac.jp 